\title{
Abordagem holística durante a gestação: alterações sistêmicas e suas repercussões na saúde bucal
}

Holistic approach during pregnancy: systemic impairments and their impact on oral health

Enfoque holístico durante el embarazo: deficiencias sistémicas y su impacto en la salud bucal Gerson Aparecido FORATORI-JUNIOR ${ }^{1}$ Priscilla Ramos PEREIRA2

${ }^{1}$ Departamento de Odontopediatria, Ortodontia e Saúde Coletiva, Faculdade de Odontologia de Bauru, USP Universidade de São Paulo, 17012-901 Bauru - SP, Brasil

${ }^{2}$ Faculdade de Odontologia, UNIFIO Centro Universitários da Faculdades Integradas de Ourinhos, 19909-100 Ourinhos - SP, Brasil

\section{Resumo}

Diversas alterações fisiológicas, imunológicas e hormonais ocorrem durante a gestação e quando associadas aos comprometimentos sistêmicos materno podem ter efeito sinérgico na saúde bucal das gestantes. O objetivo deste trabalho foi sintetizar as evidências científicas atuais de maior qualidade acerca da associação entre alterações sistêmicas e saúde bucal durante a gravidez. No período de agosto/2020 a março/2021, as seguintes bases de dados foram acessadas: EMBASE, SCOPUS, MEDLINE (Entrez PubMed), Web of Science, BVS/LILACS, e SCIELO. Foram incluídos estudos publicados entre janeiro/2000 a março/2021 em espanhol, inglês e/ou português. Devido às alterações imunológicas e hormonais da gravidez, a gengivite e a periodontite são as doenças bucais de maior prevalência nesse período. As alterações sistêmicas durante a gestação que comumente se associam à prejuízos na cavidade bucal são: anemia, sobrepeso/obesidade, ganho ponderal gestacional excessivo, diabetes mellitus e hipertensão arterial. Ademais, os acometimentos sistêmicos e periodontais na gestação podem prejudicar a saúde dos recém-nascidos. Entretanto, a obesidade e periodontite, apesar de estarem associadas, podem apresentar efeitos antagônicos na saúde dos recém-nascidos, visto que a obesidade se associa a macrossomia e a periodontite materna se associa à prematuridade e ao baixo peso ao nascer. Diante do exposto, conclui-se que diversas alterações sistêmicas se associam à piores parâmetros de saúde bucal durante a gravidez e esses fatores, conjuntamente ou isolados, podem resultar em efeitos perinatais adversos, afetando a saúde das mães e dos bebês.

Descritores: Gengivite; Gravidez; Periodontite; Saúde Bucal.

\section{Abstract}

Several physiological, immunological and hormonal changes occur during pregnancy and when they are associated with maternal systemic impairments, they may have a synergistic effect on the oral health of pregnant women. This study aimed to synthesize the current scientific evidence of higher quality about the association between systemic impairments and oral health during pregnancy. From August/2020 to March/2021, the following databases were accessed: EMBASE, SCOPUS, MEDLINE (Entrez PubMed), Web of Science, BVS/LILACS and SCIELO. Studies published between January/2000 to March/2021 in Spanish, English and/or Portuguese were included. Due to the immunological and hormonal changes of pregnancy, gingivitis and periodontitis are the most prevalent oral diseases during this period. Systemic impairments during pregnancy that are commonly associated with damage to the oral cavity are: anemia, overweight/obesity, excessive gestational weight gain, diabetes mellitus and arterial hypertension. In addition, systemic and periodontal disorders in pregnancy may harm the health of newborns. However, obesity and periodontitis, despite being associated, may have antagonistic effects on the health of newborns, since obesity is associated with macrosomia and maternal periodontitis is associated with prematurity and low birth weight. In conclusion, several systemic impairments are associated with the worst parameters of oral health during pregnancy and these factors, together or isolated, may result in adverse perinatal outcomes, affecting mothers' and babies' health.

Descriptors: Gingivitis; Pregnancy; Periodontitis; Oral Health.

\section{Resumen}

Varias alteraciones fisiológicas, inmunológicas y hormonales ocurren durante el embarazo y cuando están asociadas con alteraciones sistémicas maternas, pueden tener un efecto sinérgico sobre la salud bucal de la mujer embarazada. Este estudio tuvo como objetivo sintetizar la evidencia científica actual de mayor calidad sobre la asociación entre las alteraciones sistémicas y la salud bucal durante el embarazo. Desde agosto/2020 hasta marzo/2021, se accedió a las siguientes bases de datos: EMBASE, SCOPUS, MEDLINE (Entrez PubMed), Web of Science, BVS/LILACS y SCIELO. Se incluyeron estudios publicados entre enero/2000 a marzo/2021 en español, inglés y/o portugués. Debido a las alteraciones inmunológicos y hormonales del embarazo, la gingivitis y la periodontitis son las enfermedades bucales más prevalentes durante este período. Las alteraciones sistémicas durante el embarazo que se asocian comúnmente con daño a la cavidad oral son: anemia, sobrepeso/obesidad, aumento de peso gestacional excesivo, diabetes mellitus e hipertensión arterial. Además, los trastornos sistémicos y periodontales del embarazo pueden dañar la salud de los recién nacidos. No obstante, la obesidad y la periodontitis, a pesar de estar asociadas, pueden tener efectos antagónicos sobre la salud del recién nacido, ya que la obesidad se asocia con macrosomía y la periodontitis materna se asocia con nacimiento prematuro y bajo peso al nacer. En conclusión, varias deficiencias sistémicas están asociadas con los peores parámetros de la salud bucal durante el embarazo y estos factores, juntos o aislados, pueden producir resultados perinatales adversos que afecten la salud de las madres y los bebés.

Descriptores: Gingivitis; Embarazo; Periodontitis; Salud Bucal.

INTRODUÇÃO

De acordo com os conceitos da epigenética, o ambiente intrauterino é apontado como o primeiro contribuinte para a expressão de genes relacionados a doenças crônicas degenerativas na fase adulta ${ }^{1}$. Devido, principalmente, aos elevados níveis de estrogênio e progesterona associados à redução da atividade antimicrobiana dos neutrófilos periféricos, as mulheres durante a gestação são mais propensas à inflamação aguda nos tecidos periodontais ${ }^{2}$. Desta forma, é comum identificarmos inflamação exacerbada nos tecidos periodontais das gestantes mesmo na presença de pequena quantidade de biofilme sobre os dentes.

A periodontite na gestação pode produzir a bacteremia recorrente que, indiretamente, aciona uma fase de resposta hepática aguda, 
resultando na produção de citocinas, prostaglandinas (PGE 2) e interleucinas (IL-6 e IL-8), as quais impedem a gestação saudável, podendo afetar a saúde da gestante e do bebêt $\hat{e}^{3,4}$.

Algumas alterações sistêmicas durante a gestação, como a hipertensão, o diabetes mellitus e excesso de peso também podem trazer prejuízos para a mulher e para o bebê. Ademais essas alterações também podem contribuir para o agravamento da inflamação nos tecidos periodontais ${ }^{5-7}$.

A literatura também aponta que o maior impacto na qualidade de vida durante a gestação esteve associado a pior condição periodontal $^{8-14}$, aos dentes ausentes ${ }^{14-16}$, ao menor acesso aos serviços odontológicos ${ }^{15}$ e à maior idade das pacientes ${ }^{13,15,16}$. Desta forma, é de suma importância que os profissionais que atuam na prática odontológica conheçam as associações entre as alterações sistêmicas e bucais durante a gestação e, a partir desse conhecimento, adotem o melhor protocolo de atendimento para o pré-natal odontológico. $\mathrm{O}$ objetivo deste trabalho foi sintetizar as evidências científicas atuais de maior qualidade acerca da associação entre alterações sistêmicas e saúde bucal durante a gravidez. MATERIAL E MÉTODO

\section{- Paciente e Envolvimento Público}

O presente estudo trata-se de uma análise de dados secundários, por meio de uma revisão de literatura do tipo narrativa, uma vez que compõe uma análise da literatura publicada sobre o cuidado holístico durante a gestação. Desta forma, esta revisão de literatura foi realizada sem envolvimento do paciente. Os pacientes não foram envolvidos de forma alguma durante a revisão da literatura disponível, nem durante a redação ou edição deste documento.

- Estratégia de busca

No período de agosto/2020 a março/2021, as seguintes bases de dados foram acessadas: EMBASE (https://www.embase.com), SCOPUS (https://www.scopus.com), MEDLINE (Entrez PubMed, https://www.ncbi.nim.nih.gov), Web of Science BVS/LILACS (https://lilacs.bvsalud.org) e SCIELO (https://scielo.org), considerando as seguintes palavras-chave: (pregnancy OR pregnant woman) AND (oral health OR dental caries OR dental erosion OR gingivitis OR periodontitis OR chronic periodontitis OR aggressive periodontitis) AND (overweight OR obesity OR obesity, maternal) AND (systemic disorders OR gestational diabetes mellitus OR arterial hypertension OR anemia), as quais foram ajustadas para cada uma das bases de dados. Os respectivos termos das palavraschave citadas anteriormente em português e espanhol também foram acessados.

\section{- Critérios de elegibilidade}

Foram selecionados nesta revisão de literatura estudos publicados entre janeiro/2000 e março/2021, nos idiomas português, inglês e espanhol. Dentre eles, foram considerados: clínicos intervencionais (ensaios clínicos randomizados e não randomizados; e não-cego, duplo-cego ou triplo-cego) e observacionais (transversais, coortes e de caso-controle). Outras revisões de literatura (sistemática com e sem meta-análise; ou não sistemática) foram incluídas. Além disso, foram considerados relatórios governamentais (Organização Mundial da Saúde e Ministério da Saúde) e protocolos institucionais (Instituto de Medicina). Não foram considerados nesta revisão de literatura os relatos de caso, séries de casos, artigos de opinião, editoriais, cartas aos editores, estudos laboratoriais envolvendo animais, protocolos metodológicos e resumos de eventos científicos (expandidos ou não expandidos).

RESULTADOS E DISCUSSÃO

A gestação é marcada por várias alterações no corpo da mulher, as quais acontecem para manutenção do corpo em todo o período gestacional e também para o desenvolvimento do feto. Dentro dessas alterações podemos citar as fisiológicas, imunológicas e as hormonais ${ }^{2}$.

Estudos mostram que ao longo da gestação ocorre um aumento das bactérias aeróbias e anaeróbias nos tecidos gengivais das mulheres em decorrência do aumento nos níveis de estrogênio e progesterona no corpo das mulheres ${ }^{17}$. Ademais, frente as alterações fisiológicas durante a gestação, existe um prejuízo no turnover do tecido conjuntivo no periodonto, podendo resultar no aumento da resposta inflamatória nos tecidos periodontais ${ }^{2}$.

Durante a gestação, existem também diversas alterações sistêmicas que podem prejudicar os desfechos perinatais, dentre elas, podem ser citadas: a anemia, o excesso de peso materno, o ganho ponderal excessivo na gravidez, o diabetes mellitus e a hipertensão arterial| $^{5-7,18,19}$.

É importante ressaltar que nem todas as gestantes apresentam tais alterações biológicas acima citadas. A condição de saúde das pacientes está intimamente associação aos Determinantes Sociais de Saúde (DSS).

Os Determinantes Socais de Saúde são características específicas do contexto social 
que de alguma maneira pode afetar a saúde, por meio de condições sociais, como renda, educação, ocupação, estrutura familiar, disponibilidade de serviços, saneamento, exposições às doenças, redes e apoio social, discriminação social e acesso às ações preventivas de saúde ${ }^{20}$. Com o avanço do conhecimento a respeito dos DSS modelos que destacam as implicações das iniquidades sociais na saúde foram criados. O modelo de Dahlgren e Whitehead foi o adotado pela Comissão Nacional sobre os Determinantes Sociais da Saúde (CNDSS) por sua compreensão ser mais fácil e transparente. Este modelo explica o mecanismo pelo qual a interação entre diferentes níveis de condições sociais leva à iniquidade em saúde, desde o nível individual até 0 nível das condições econômicas, culturais e ambientais dominantes em toda sociedade.

Considerando, portanto, que os Determinantes Sociais de Saúde influenciam a condição de vida dos indivíduos a depender do contexto ao qual se inserem, é cabível compreendermos que algumas gestantes estarão expostas a determinados fatores de risco comparadas a outras mulheres ${ }^{20}$. Assim, devemos individualizar cada contexto a fim de elaborarmos estratégias efetivas de saúde ao atendermos as mulheres durante a gestação.

- Anemia na gestação e condição bucal

O diagnóstico de anemia durante a gestação é comum devido ao aumento volumétrico do sangue, sem que ocorra 0 aumento proporcional nos níveis férricos ${ }^{18}$. A anemia por deficiência do ferro durante a gestação é um fator de risco para a incidência da cárie dentária nas mulheres na gestação ${ }^{18}$. A hipótese que melhor discorre acerca do papel protetor da presença do ferro no processo da cárie dentária evidencia que o ferro afeta a acidogenicidade $e$ inibe a atividade da glicosiltransferase dos Streptococcus mutans. Desta forma, o ferro presente inibe a atividade das bactérias que estão presentes na doença cárie e, além disso, reduz a produção dos polissacarídeos extracelulares ${ }^{18}$.

Apesar da deficiência de ferro poder se associar à maior incidência da cárie dentária, outros fatores relacionados aos hospedeiros devem ser considerados, como saliva, capacidade tampão, dieta (quantidade e frequência do consumo de açúcares) ${ }^{21}$. Ademais, é importante considerar os fatores extrínsecos, como os fatores sociais, econômicos e culturais ${ }^{21}$. Portanto, é essencial que o profissional conheça o contexto de cada paciente principalmente no período gestacional, para que se obtenha sucesso na intervenção.

Considerando o diagrama de Keyes Modificado acerca dos fatores causais da cárie dentária, sabe-se que o tempo é um dos fatores causais para a instalação e desenvolvimento da doença. Desta forma, ainda que durante a gestação as mulheres possam apresentar mudanças comportamentais de higiene e dieta $^{22}$, contribuindo com o aumento no risco da doença cárie, não há evidência plausível de que as lesões cariosas observadas em mulheres grávidas são, de fato, resultantes do período gestacional, o qual é caracterizado pelo período aproximado de 40 semanas. Desta forma, é importante considerar que a progressão da doença cárie possa ser resultante de um processo prévio a gestação.

- Sobrepeso/obesidade na gestação e condição bucal

A obesidade é uma doença crônica e inflamatória, que está relacionada aos fatores genéticos, metabólicos, culturais e comportamentais $^{23,24}$. O excesso de peso associa-se a efeitos adversos na gestação como a hipertensão arterial, pré-eclâmpsia, diabetes mellitus gestacional (DMG), tamanho excessivo do bebê para a idade gestacional, parto prematuro, macrossomia, cesariana de emergência e parto forçado com auxílio de instrumentais $^{25-29}$. O sobrepeso e a obesidade durante a gestação também estão relacionados ao maior risco de obesidade e complicações metabólicas e cardiovasculares para o filho ${ }^{30,31}$.

O tecido adiposo de pacientes obesos secreta citocinas inflamatórias e adipocinas, como TNF- $\alpha$, IL-6, adiponectina, leptina, resistina, os quais reduzem a resposta imune do hospedeiro e causam um estado inflamatório generalizado do corpo ${ }^{32-34}$. Portanto, na presença de placa bacteriana o paciente com obesidade terá uma resposta inflamatória dos tecidos periodontais mais exacerbada. Fortes evidências científicas têm demonstrado a associação positiva entre a obesidade e a periodontite ${ }^{32-34}$.

A obesidade materna e a gestação agem de forma sinérgica nos tecidos periodontais. Portanto, frente aos elevados níveis hormonais associados aos mediadores inflamatórios que são secretados pelo tecido adiposo de mulheres com excesso de peso, é esperado que gestantes com sobrepeso/obesidade apresentem uma pior condição periodontal ${ }^{5,8,9,35-42}$.

- Ganho de peso excessivo na gestação e condição bucal 
Além da análise do estado nutricional da mulher, outro fator que deve ser avaliado é o ganho ponderal de acordo com a idade gestacional. Estudos apontam que o ganho de peso excessivo está ligado a uma série de complicações durante a gravidez e durante o parto em si, como a DMG, hipertensão, préeclâmpsia e aborto espontâne ${ }^{30}$. Cesariana de emergência, parto prematuro e de natimorto são outros exemplos. Os filhos de pacientes com ganho de peso excessivo têm maior probabilidade de ter defeitos congênitos e de morrer durante o nascimento ou logo depois dele. Os que sobrevivem têm grandes chances de desenvolver hipertensão e obesidade na idade adulta ${ }^{43}$.

De acordo com o Instituto de Medicina norte-americano, o ganho ponderal durante todo o período gestacional está diretamente ligado ao estado nutricional prévio de cada mulher ${ }^{44}$. A tabela 1 detalha a recomendação do ganho ponderal ao longo de toda gestação de acordo com a classificação do estado nutricional prégestacional.

Tabela 1. Ganho ponderal recomendado de acordo com IMC materno pré-gestacional $^{44}$

\begin{tabular}{c|c|c}
\hline $\begin{array}{c}\text { Estado nutricional } \\
\text { antes da gestação }\end{array}$ & IMC $\left(\mathbf{k g} / \mathbf{m}^{2}\right)$ & $\begin{array}{c}\text { Ganho de peso } \\
\text { durante a gestação } \\
(\mathbf{k g})\end{array}$ \\
\hline Baixo peso & $<18,5$ & $12,5-18$ \\
\hline Peso adequado & $18,5-24,9$ & $11-16$ \\
\hline Sobrepeso & $25,0-29,9$ & $7-11,5$ \\
\hline Obesidade & $\geq 30,0$ & $5-9$ \\
\hline Fonte: IOM - Institute of Medicine ${ }^{44}$ &
\end{tabular}
Jesuino et al. $^{7}$ avaliaram a condição sistêmica e periodontal de mulheres que apresentaram ganho ponderal gestacional excessivo. Os autores destacaram que gestantes com ganho ponderal excessivo apresentam alta prevalência de hipertensão e periodontite, sendo que a periodontite persiste mesmo após o parto. Ademais, o ganho de peso excessivo na gestação se associou significativamente ao alto IMC dos recémnascidos ${ }^{7}$.

\section{- Diabetes mellitus gestacional (DMG) e condição bucal}

DMG é causado pela redução da função pancreática das células- $\beta$ e está associado ao aumento nos efeitos perinatais adversos, aumento do risco de obesidade nos filhos e elevado risco de desenvolvimento de diabetes mellitus nas mães ${ }^{45}$. As gestantes que apresentam DMG podem ser tratadas através do uso de insulina ou agentes antidiabéticos, quando os níveis de glicose materna e/ou parâmetros de crescimento fetal indicarem risco de complicações perinatais. $O$ acompanhamento em longo prazo das mães inclui a avaliação do nível e tipo do risco de diabetes, estilo de vida e/ou abordagens farmacológicas. Para os filhos de gestantes com
DMG o acompanhamento deve se concentrar na detecção e na redução do risco de desenvolvimento da obesidade e suas complicações $^{30,46}$.

Evidências prévias mostraram a associação do DMG com a periodontite. ${ }^{47,48} \mathrm{~A}$ relação entre esses desfechos permanece discutível e pode ser mal interpretada por uma variedade de fatores de confusão. É possível, ainda, que a periodontite seja o fator etiológico do DMG e não uma consequência, uma vez que a inflamação crônica subclínica da periodontite induz respostas imunológicas locais do hospedeiro e causa bacteremia transitória, que afeta a saúde sistêmica do indivíduo ${ }^{49}$. A inflamação gengival materna pode resultar em resistência à insulina, o que pode exacerbar a resistência à insulina e diminuir a tolerância à glicose e, consequentemente, levar ao $\mathrm{DMG}^{50}$.

- Hipertensão arterial na gestação e condição bucal

A hipertensão induzida pela gravidez é definida como 0 desenvolvimento de hipertensão arterial durante a gravidez, com valores iguais ou superiores a $140 / 90 \mathrm{mmHg}$, normalizando essa condição após 0 parto. ${ }^{51}$ Estudos mostram maior risco de resultados adversos da gravidez entre mulheres com hipertensão induzida pela gravidez em comparação com mulheres normotensas, como: descolamento da placenta, eventos cerebrovasculares, falência de órgãos e coagulação intravascular disseminada. Além disso, os fetos dessas mães apresentam maior risco de retardo de crescimento intrauterino, prematuridade e morte intrauterina. ${ }^{51,52}$

Atualmente, evidências científicas têm mostrado a associação entre hipertensão arterial e periodontite. ${ }^{53,54}$ No entanto, a relação casual entre esses desfechos não é clara, principalmente devido ao fato destas condições serem moduladas por diversos outros fatores e, também, devido à existência de diversas classificações para as doenças periodontais, que prejudica a comparação entre os estudos. Pralhad e colaboradores ${ }^{55}$ (2013) concluíram que a doença periodontal é prevalente na gravidez e que há uma tendência palpável de aumento da gravidade da hipertensão à medida que a doença periodontal piora.

- Periodontite materna e saúde do bebê ao nascer

Evidências prévias associaram a periodontite na gestação com a prematuridade e com o baixo peso ao nascer ${ }^{3,4}$. Existem dois mecanismos plausíveis que explicam essa associação: 1) as bactérias periodontopatogênicas desencadeiam uma 
cascata de inflamação nos tecidos periodontais, desta forma, citocinas pró-inflamatórias decorrentes da destruição dos tecidos periodontais atingem a corrente sanguínea e mediam uma inflamação na placenta, de maneira indireta, prejudicando 0 desenvolvimento normal do feto; 2) A via direta é quando as próprias bactérias decorrentes das doenças periodontais se alojam, via corrente sanguínea, na placenta, desencadeando inflamação no local e prejudicando o desenvolvimento fetal ${ }^{3,4}$.

- Obesidade, periodontite e saúde do bebê ao nascer

A obesidade materna e a periodontite durante a gestação agem de maneira antagônica nos parâmetros de saúde do bebê ao nascer, impactando negativamente na qualidade de vida das mulheres ${ }^{8,9}$. Enquanto a periodontite na gestação pode resultar em prematuridade e baixo peso ao nascer ${ }^{3,4}$, a obesidade está associada à macrossomia ${ }^{56}$, que é o nascimento de crianças com peso e estatura acima do que são considerados normais. O efeito antagônico desses desfechos torna-se uma incoerência científica a ser estudada.

Foratori-Junior et al. ${ }^{42}$ avaliaram a associação entre 0 excesso de peso prégestacional, a periodontite e o peso do bebê ao nascer. Os autores destacaram que o peso excessivo antes da gravidez esteve significativamente associado a hipertensão arterial, diabetes mellitus e periodontite durante o terceiro trimestre da gestação. Além disso, o sobrepeso/obesidade esteve associado a um peso baixo/insuficiente ao nascer ${ }^{42}$. Possivelmente, a periodontite materna foi determinante para o nascimento de bebês com peso abaixo do normal. No entanto, os autores destacam que estudos futuros longitudinais devem ser realizados a fim de melhor compreender a associação desses desfechos.

CONCLUSÃO

Devido às alterações imunológicas e hormonais da gravidez, a gengivite e a periodontite são as doenças bucais de maior prevalência nesse período. Os acometimentos sistêmicos e periodontais na gestação podem prejudicar a saúde dos recém-nascidos. As alterações sistêmicas durante a gestação que mais comumente se associam à prejuízos na saúde bucal são: anemia, sobrepeso/obesidade, ganho ponderal gestacional excessivo, diabetes mellitus e hipertensão arterial. Desta forma, os profissionais de saúde devem atuar de maneira interdisciplinar durante o pré-natal, a fim de garantir saúde a elas e aos bebês.
REFERENCIAS

1. Karin-Kujundzic V, Sola IM, Predavec $N$, Potkonjak A, Somen E, Mioc P, et al. Novel Epigenetic Biomarkers in Pregnancy-Related Disorders and Cancers. Cells. 2019;8:1459.

2. Silva de Araujo Figueiredo C, Gonçalves Carvalho Rosalem C, Costa Cantanhede AL, Abreu Fonseca Thomaz ÉB, Fontoura Nogueira da Cruz MC. Systemic alterations and their oral manifestations in pregnant women. J Obstet Gynaecol Res. 2017;43:16-22.

3. Manrique-Corredor EJ, Orozco-Beltran $D$, Lopez-Pineda A, Quesada JA, Gil-Guillen VF, Carratala-Munuera C. Maternal periodontitis and preterm birth: Systematic review and metaanalysis. Community Dent Oral Epidemiol. 2019;47:243-51.

4. Figuero E, Han YW, Furuichi Y. Periodontal diseases and adverse pregnancy outcomes: Mechanisms. Periodontol 2000. 2020;83: 175-88.

5. Foratori-Junior GA, da Silva BM, da Silva Pinto AC, Honório HM, Groppo FC, de Carvalho Sales-Peres SH. Systemic and periodontal conditions of overweight/obese patients during pregnancy and after delivery: a prospective cohort. Clin Oral Investig. 2020;24:157-65.

6. Genco RJ, Graziani F, Hasturk H. Effects of periodontal disease on glycemic control, complications, and incidence of diabetes mellitus. Periodontol 2000. 2020;83:59-65.

7. Jesuino BG, Foratori-Junior GA, Missio ALT, Mascoli LS, Sales-Peres SHC. Periodontal status of women with excessive gestational weight gain and the association with their newborns' health. Int Dent J. 2020;70:396-404.

8. Caracho RA, Foratori-Junior GA, Fusco NDS, Jesuino BG, Missio ALT, Sales-Peres SHC. Systemic conditions and oral health-related quality of life of pregnant women of normal weight and who are overweight. Int Dent $\mathrm{J}$. 2020;70:287-95.

9. Foratori-Junior GA, Missio ALT, Orenha ES, de Carvalho Sales-Peres SH. Systemic Condition, Periodontal Status, and Quality of Life in Obese Women During Pregnancy and After Delivery. Int Dent J. 2021;71:420-8.

10. Acharya S, Bhat PV. Oral-health-related quality of life during pregnancy. J Public Health Dent. 2009;69:74-7.

11. Acharya S, Bhat PV, Acharya S. Factors affecting oral health-related quality of life among pregnant women. Int J Dent Hyg. 2009; 7:102-7.

12. Geevarghese A, Baskaradoss JK, Sarma PS. Oral Health-Related Quality of Life and Periodontal Status of Pregnant Women. Matern Child Health J. 2017;21:1634-42.

13. Shah AF, Batra M, Qureshi A. Evaluation of impact of pregnancy on oral health status and 
oral health related quality of life among women of Kashmir Valley. J Clin Diagn Res. 2017;11:ZC01-ZC04. Retraction in: J Clin Diagn Res. 2018;12:ZZ01.

14. Wandera MN, Engebretsen IM, Rwenyonyi CM, Tumwine J, Astrøm AN; PROMISE-EBF Study Group. Periodontal status, tooth loss and selfreported periodontal problems effects on oral impacts on daily performances, OIDP, in pregnant women in Uganda: a cross-sectional study. Health Qual Life Outcomes. 2009;7:89.

15. Lu HX, Xu W, Wong MC, Wei TY, Feng XP. Impact of periodontal conditions on the quality of life of pregnant women: a cross-sectional study. Health Qual Life Outcomes. 2015;13:67.

16. Moimaz SA, Rocha NB, Garbin AJ, Garbin CA, Saliba $O$. Influence of oral health on quality of life in pregnant women. Acta Odontol Latinoam. 2016;29:186-93.

17. Reis DM, Pitta DR, Ferreira HM, de Jesus MC, de Moraes ME, Soares MG. Educação em saúde como estratégia de promoção de saúde bucal em gestantes. Cienc saúde coletiva. 2010;15:269-76.

18. Costa EM, Azevedo JAP, Martins RFM, Alves CMC, Ribeiro CCC, Thomaz EBAF. Anemia and Dental Caries in Pregnant Women: a Prospective Cohort Study. Biol Trace Elem Res. 2017;177:241-50.

19. Oskovi-Kaplan ZA, Ozgu-Erdinc AS. Management of Gestational Diabetes Mellitus. Adv Exp Med Biol. 2021;1307:257-72.

20. Geib LTC. Determinantes sociais da saúde do idoso. Cienc saude coletiva. 2012;17:123-33.

21. Selwitz RH, Ismail Al, Pitts NB. Dental caries. Lancet. 2007;369:51-9.

22. Kateeb E, Momany E. Dental caries experience and associated risk indicators among Palestinian pregnant women in the Jerusalem area: a cross-sectional study. BMC Oral Health. 2018;18:170.

23. Foratori GA Junior, Andrade FJ, Mosquim V, Sales Peres MC, Ceneviva R, Chaim EA, Sales Peres SH. Presence of Serum Ferritin before and after Bariatric Surgery: Analysis in Dentate and Edentulous Patients. PLoS One. 2016;11:e0164084.

24. World Health Organization. Obesity: Preventing and managing the global epidemic. Geneva: World Health Organization; 2000. p. 1-252. Available

from: http://www.who.int/nutrition/publications/obesity/ WHO_TRS_894/en/.

25. Yu Z, Han S, Zhu J, Sun X, Ji C, Guo X. Prepregnancy body mass index in relation to infant birth weight and offspring overweight/obesity: a systematic review and meta-analysis. PLoS One. 2013;8:e61627.

26. Li G, Kong L, Li Z, Zhang L, Fan L, Zou L, et al. Prevalence of macrosomia and its risk factors in china: a multicentre survey based on birth data involving 101,723 singleton term infants. Paediatr Perinat Epidemiol. 2014;28:345-50.

27. El Rafei R, Abbas HA, Charafeddine L, Nakad P, Al Bizri A, Hamod D, et al. Association of pre-pregnancy body mass index and gestational weight gain with preterm births and fetal size: an observational study from Lebanon. Paediatr Perinat Epidemiol. 2016;30:38-45.

28.Zilberlicht A, Feferkorn I, Younes G, Damti A, Auslender R, Riskin-Mashiah S. The mutual effect of pregestational body mass index, maternal hyperglycemia and gestational weight gain on adverse pregnancy outcomes. Gynecol Endocrinol. 2016;32:416-20.

29. Gao X, Yan Y, Xiang S, Zeng G, Liu S, Sha T, et al. The mutual effect of pre-pregnancy body mass index, waist circumference and gestational weight gain on obesity-related adverse pregnancy outcomes: A birth cohort study. PLoS One. 2017;12:e0177418.

30. Buchanan TA, Xiang AH, Page KA. Gestational diabetes mellitus: risks and management during and after pregnancy. Nat Rev Endocrinol. 2012;8:639-49.

31. Tan HC, Roberts J, Catov J, Krishnamurthy R, Shypailo R, Bacha F. Mother's pre-pregnancy $\mathrm{BMI}$ is an important determinant of adverse cardiometabolic risk in childhood. Pediatr Diabetes. 2015;16:419-26.

32. Moura-Grec PG, Marsicano JA, Carvalho CA, Sales-Peres SH. Obesity and periodontitis: systematic review and meta-analysis. Cien Saude Colet. 2014;19:1763-72.

33. Keller A, Rohde JF, Raymond K, Heitmann BL. Association between periodontal disease and overweight and obesity: a systematic review. J Periodontol. 2015;86:766-76.

34. Khan S, Barrington G, Bettiol S, Barnett T, Crocombe $L$. Is overweight/obesity a risk factor for periodontitis in young adults and adolescents?: a systematic review. Obes Rev. 2018;19:852-83.

35. Chapper A, Munch A, Schermann C, Piacentini CC, Fasolo MT. Obesity and periodontal disease in diabetic pregnant women. Braz Oral Res. 2005;19:83-7.

36. Piscoya MD, Ximenes RA, Silva GM, Jamelli SR, Coutinho SB. Periodontitis-associated risk factors in pregnant women. Clinics (Sao Paulo). 2012;67:27-33.

37. Vogt M, Sallum AW, Cecatti JG, Morais SS. Factors associated with the prevalence of periodontal disease in low-risk pregnant women. Reprod Health. 2012;9:3.

38. Lee HJ, Jun JK, Lee SM, Ha JE, Paik DI, Bae $\mathrm{KH}$. Association between obesity and periodontitis in pregnant females. J Periodontol. 2014;85:e224-31. 
39. Xie Y, Xiong X, Elkind-Hirsch KE, Pridjian G, Maney $\mathrm{P}$, Delarosa $\mathrm{RL}$, et al. Prepregnancy obesity and periodontitis among pregnant females with and without gestational diabetes mellitus. J Periodontol. 2014;85:890-8.

40.Zambon M, Mandò C, Lissoni A, Anelli GM, Novielli C, Cardellicchio M, et al. Inflammatory and Oxidative Responses in Pregnancies With Obesity and Periodontal Disease. Reprod Sci. 2018;25:1474-84.

41. Fusco NDS, Foratori-Junior GA, Missio ALT, Jesuino BG, Sales-Peres SHC. Systemic and oral conditions of pregnant women with excessive weight assisted in a private health system. Int Dent J. 2019;69:472-9.

42. Foratori-Junior GA, Jesuino BG, Caracho RA, Orenha ES, Groppo FC, Sales-Peres SHC. Association between excessive maternal weight, periodontitis during the third trimester of pregnancy, and infants' health at birth. J Appl Oral Sci. 2020;28:e20190351.

43. Rath SR, Marsh JA, Newnham JP, Zhu K, Atkinson $\mathrm{HC}$, Mountain J, et al. Parental prepregnancy $\mathrm{BMI}$ is a dominant early-life risk factor influencing BMI of offspring in adulthood. Obes Sci Pract. 2016;2:48-57.

44. Institute of Medicine (US) and national research council (US) committee to reexamine IOM pregnancy weight guidelines. Weight gain during pregnancy: reexamining the guidelines. Washington: The National Academies Collection: Reports funded by National Institutes of Health, 2009.

45. Catalano PM. Obesity, insulin resistance, and pregnancy outcome. Reproduction. 2010;140:365-71.

46. Artal R, Catanzaro RB, Gavard JA, Mostello DJ, Friganza JC. A lifestyle intervention of weightgain restriction: diet and exercise in obese women with gestational diabetes mellitus. Appl Physiol Nutr Metab. 2007;32:596-601.

47. Abariga SA, Whitcomb BW. Periodontitis and gestational diabetes mellitus: a systematic review and meta-analysis of observational studies. BMC Pregnancy Childbirth. 2016;16:344.

48. Kumar A, Sharma DS, Verma M, Lamba AK, Gupta MM, Sharma S, et al. Association between periodontal disease and gestational diabetes mellitus-A prospective cohort study. $\mathrm{J}$ Clin Periodontol. 2018;45:920-31.

49. Xiong $X$, Elkind-Hirsch KE, Vastardis $S$, Delarosa RL, Pridjian G, Buekens $P$. Periodontal disease is associated with gestational diabetes mellitus: a case-control study. J Periodontol 2009;80:1742-9.

50. Özçaka Ö, Ceyhan-Öztürk $B$, Gümüş $P$, Akcalı A, Nalbantsoy A, Buduneli N. Clinical periodontal status and inflammatory cytokines in gestational diabetes mellitus. Arch Oral Biol 2016;11:87-91.

51.Kintiraki E, Papakatsika S, Kotronis G, Goulis DG, Kotsis V. Pregnancy-Induced hypertension. Hormones (Athens). 2015;14:211-23.

52. Berhe AK, llesanmi AO, Aimakhu CO, Mulugeta A. Effect of pregnancy induced hypertension on adverse perinatal outcomes in Tigray regional state, Ethiopia: a prospective cohort study. BMC Pregnancy Childbirth. 2019;20:7.

53. Martin-Cabezas R, Seelam N, Petit C, Agossa $\mathrm{K}$, Gaertner S, Tenenbaum H, et al. Association between periodontitis and arterial hypertension: A systematic review and meta-analysis. Am Heart J. 2016;180:98-112.

54. Muñoz Aguilera E, Suvan J, Buti J, Czesnikiewicz-Guzik M, Barbosa Ribeiro A, Orlandi $\mathrm{M}$, et al. Periodontitis is associated with hypertension: a systematic review and metaanalysis. Cardiovasc Res. 2020;116:28-39.

55. Pralhad S, Thomas B, Kushtagi P. Periodontal disease and pregnancy hypertension: a clinical correlation. J Periodontol. 2013;84:1118-25.

56. Zhao R, Xu L, Wu ML, Huang SH, Cao XJ. Maternal pre-pregnancy body mass index, gestational weight gain influence birth weight. Women Birth. 2018;31:e20-e25.

\section{CONFLITO DE INTERESSES}

Os autores declaram não haver conflitos de interesse AUTOR PARA CORRESPONDÊNCIA

\section{Prof. Me. Gerson Aparecido Foratori-Junior}

Departamento de Odontopediatria, Ortodontia e Saúde Coletiva,

Faculdade de Odontologia de Bauru,

Universidade de São Paulo, USP

Bauru - SP, Brasil

Tel: (11) 99766-7202

Email: gerson.foratori@usp.br
Submetido em 22/04/2021 Aceito em 16/07/2021 